\title{
Correlation between the latent heats and cohesive energies of metal clusters
}

\author{
Anne K. Starace ${ }^{1}$ Colleen M. Neal, ${ }^{1}$ Baopeng Cao, ${ }^{1}$ Martin F. Jarrold, ${ }^{1, a)}$ \\ Andrés Aguado, ${ }^{2, b)}$ and José M. López ${ }^{2}$ \\ ${ }^{1}$ Chemistry Department, Indiana University, 800 East Kirkwood Ave., Bloomington, Indiana 47405, USA \\ ${ }^{2}$ Departamento de Física Teórica, Universidad de Valladolid, Valladolid 47011, Spain
}

(Received 10 June 2008; accepted 27 August 2008; published online 9 October 2008)

\begin{abstract}
Dissociation energies have been determined for $\mathrm{Al}_{n}{ }^{+}$clusters $(n=25-83)$ using a new experimental approach that takes into account the latent heat of melting. According to the arguments presented here, the cohesive energies of the solidlike clusters are made up of contributions from the dissociation energies of the liquidlike clusters and the latent heats for melting. The size-dependent variations in the measured dissociation energies of the liquidlike clusters are small and the variations in the cohesive energies of solidlike clusters result almost entirely from variations in the latent heats for melting. To compare with the measured cohesive energies, density-functional theory has been used to search for the global minimum energy structures. Four groups of low energy structures were found: Distorted decahedral fragments, fcc fragments, fcc fragments with stacking faults, and "disordered." For most cluster sizes, the measured and calculated cohesive energies are strongly correlated. The calculations show that the variations in the cohesive energies (and the latent heats) result from a combination of geometric and electronic shell effects. For some clusters an electronic shell closing is responsible for the enhanced cohesive energy and latent heat (e.g., $n=37$ ), while for others (e.g., $n=44)$ a structural shell closing is the cause. (C) 2008 American Institute of Physics.
\end{abstract}

[DOI: $10.1063 / 1.2987720$ ]

\section{INTRODUCTION}

As the size of an object decreases, its properties change. The change starts as a smooth evolution in the mesoscopic size regime and becomes discontinuous in the cluster size regime (fewer than around $10^{3}$ atoms). Here we are concerned with the evolution of the dissociation energy, and specifically how the energy needed to remove an atom from an object at $0 \mathrm{~K}$ changes with size. For the bulk, the dissociation energy is the same as the cohesive energy (and equivalent to the atomization energy), but for a small cluster they are not the same because the dissociation energies are size dependent.

Heating a bulk solid usually causes it to melt and then with further heating the liquid eventually boils. A heating curve, a plot of temperature against added energy, appears in most introductory chemistry texts. It shows that the latent heat of melting is part of the energy that must be added to remove an atom from the solid. The dissociation energy can be determined from the latent heat of melting, the latent heat of vaporization, and the heat capacities of the solid, liquid, and gas.

The dissociation energy of an isolated molecule or ion is usually measured by determining how much energy must be added to drive fragmentation. For small molecules and ions, fragmentation occurs promptly when enough energy has been added to exceed the dissociation threshold. However, for molecules with more than a few atoms, the energy is

\footnotetext{
${ }^{a)}$ Electronic mail: mfj@indiana.edu.

${ }^{b)}$ Electronic mail: aguado@metodos.fam.cie.uva.es
}

dispersed among the vibrational modes and additional energy must be added to speed up the fragmentation so that it occurs within the experimental time frame. The excess energy can be accounted for by a statistical model. There is no consideration of the phase of the dissociating molecule in this analysis. Most molecules have a well-defined connectivity between their constituent atoms, which does not change as they are heated to the point where they dissociate. In other words, molecules do not melt.

In the past, the dissociation energies of metal clusters have been determined using the same strategy that is used to measure the dissociation energies of isolated molecules and ions. $^{1-9}$ This is the correct approach for the very small clusters that behave like molecules and do not melt. However, it is now well established that even relatively small metal clusters can undergo a meltinglike transition with a significant latent heat. ${ }^{10-19}$ Does the latent heat need to be considered when determining the dissociation energy?

Figure 1 shows the relationship between solidlike and liquidlike clusters and the dissociation threshold. In this work we take the dissociation threshold to mean the energy required for half of the clusters to dissociate on the experimental time scale. This is different from a true threshold measurement which seeks to identify the minimum energy required for dissociation. Consider what occurs if we start with a solidlike cluster with an internal energy corresponding to the average at $300 \mathrm{~K}$. As the internal energy is increased, the clusters melt and become liquidlike. Melting is accompanied by a latent heat, $L(n)$. More energy is then added to the liquidlike cluster until it is eventually energized to the 


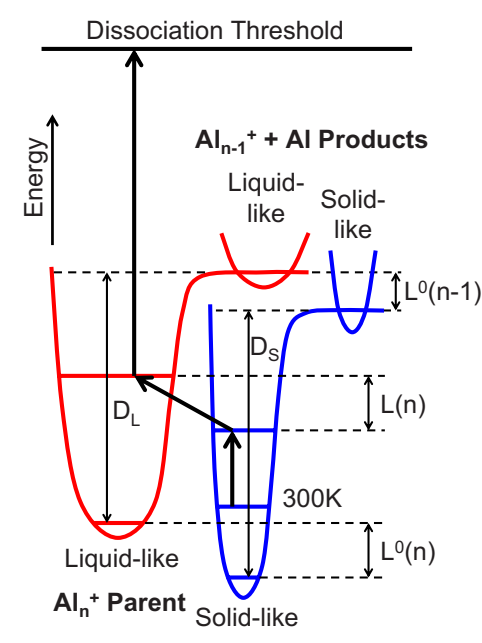

FIG. 1. (Color online) Schematic diagram showing the relationship between solidlike and liquidlike clusters and the dissociation threshold that results from the loss of a single atom from the cluster. At $300 \mathrm{~K}$ the cluster is solidlike but dissociation occurs from the liquidlike cluster. $D_{S}$ is the $0 \mathrm{~K}$ energy difference between the solidlike $\mathrm{Al}_{n}^{+}$cluster and solidlike $\mathrm{Al}_{n-1}{ }^{+}$ $+\mathrm{Al}$ products, and $D_{L}$ is the $0 \mathrm{~K}$ energy difference between liquidlike $\mathrm{Al}_{n}^{+}$ cluster and liquidlike $\mathrm{Al}_{n-1}{ }^{+}+\mathrm{Al}$ products. $L(n)$ is the latent heat for melting of the $\mathrm{Al}_{n}{ }^{+}$cluster at its melting temperature (where the number of solidlike clusters equals the number of liquidlike). $L^{0}(n)$ and $L^{0}(n-1)$ are the energy differences between the liquidlike and solidlike states at $0 \mathrm{~K}$ for the $n$ atom and $n-1$ atom clusters, respectively.

point where it dissociates. Dissociation occurs by loss of an atom and we will show below that the $n-1$ atom product cluster is liquidlike. Since dissociation occurs from the liquidlike state to give a liquidlike product, a rigorous analysis of the measured dissociation threshold (using a statistical model to account for the excess energy) yields $D_{L}$, the dissociation energy of the liquidlike cluster. The liquidlike cluster does not exist at $0 \mathrm{~K}$; however, $D_{L}$ is still a meaningful quantity in the sense that its value can be deduced by extrapolation from finite temperatures. Below, we show that the relationship between $D_{L}$ and the dissociation energy of a solidlike cluster $D_{S}$ is

$$
D_{S}(n) \approx D_{L}(n)+L(n)-L(n-1),
$$

where $L(n)$ and $L(n-1)$ are the latent heats of melting for clusters with $n$ and $n-1$ atoms, respectively. The cohesive energy (the total binding energy of the cluster divided by the number of atoms) is given by

$$
C_{S}(n)=\frac{\sum_{n} D_{S}(n)}{n} \approx \frac{L(n)+\sum_{n} D_{L}(n)}{n} .
$$

Since $\Sigma D_{L}$ is not expected to show significant sizedependent fluctuations, fluctuations in the cohesive energies of the solidlike clusters are expected to result almost entirely from fluctuations in the latent heats.

In this paper we report on dissociation energies for solidlike aluminum cluster cations with 25-83 atoms determined from dissociation thresholds for liquidlike clusters and latent heats for melting. In addition, calculations were performed at the Kohn-Sham density-functional-theory level to search for the global minimum energy structures of cluster cations with 34-83 atoms. For most cluster sizes, the fluctuations in the calculated cohesive energies are strongly cor- related with fluctuations in the experimental values. The calculations provide insight into the physical factors that cause some clusters to have enhanced cohesive energies (and enhanced latent heats). Finally, in order to confirm the generality of the correlation between the cohesive energies and latent heats reported here for aluminum clusters, we have tested it for sodium clusters.

\section{EXPERIMENTAL METHODS}

The dissociation thresholds are measured using multicollision induced dissociation. ${ }^{20-22}$ Aluminum cluster ions are generated by pulsed laser vaporization of a liquid metal target. ${ }^{23}$ Before they exit the source, their temperature is set to $300 \mathrm{~K}$ in a $10 \mathrm{~cm}$ long temperature-variable extension. The exiting cluster ions are focused into a quadrupole mass spectrometer where a specific cluster size is selected. The size-selected clusters are then focused into a high pressure collision cell. As the clusters enter the collision cell they undergo numerous collisions with the helium collision gas, each one converting a fraction of the ion's translational energy into internal energy and translational energy of the collision partner. If their initial translational energy is high enough, the cluster ions may be heated to the point where they dissociate. Dissociation occurs through loss of aluminum atoms. The fragment ions and undissociated clusters are focused into a second quadrupole mass spectrometer where they are analyzed. The fraction of the cluster ions that dissociate is determined from the mass spectrum. Typically six measurements were performed as a function of the initial translational energy, and the initial translational energy required to dissociate $50 \%$ of the clusters is determined from a linear regression. Four independent measurements of the dissociation thresholds were made. The average root-meansquare deviation is $0.3 \%$.

\section{ANALYSIS OF MEASURED DISSOCIATION THRESHOLDS}

In order for the clusters to dissociate in the experimental time scale they must have an internal energy much larger than their dissociation energy. We account for this excess energy using a statistical model. The fraction of the clusters that dissociate is given by

$$
f=\frac{\int_{0}^{\infty} d E_{V} \rho_{V}\left(E_{V}\right) e^{-E_{V} / k_{B} T}\left(1-e^{-k(E, D) t}\right)}{\int d E_{V} \rho_{V}\left(E_{V}\right) e^{-E_{V} / k_{B} T}},
$$

where $E_{V}$ is the initial vibrational energy of the cluster, $T$ is the temperature of the source extension, $t$ is the time that the clusters remain hot, and $\rho_{V}$ is the vibrational density of states. $k(E, D)$ is the rate constant for unimolecular dissociation of a cluster with dissociation energy $D$ and total internal energy $E$. In this work, we use the quantum RiceRamsperger-Kassel (RRK) model ${ }^{24}$ for the reaction rate constant,

$$
k(E, D)=g \nu \frac{p !(p-q+s-1) !}{(p+s-1) !(p-q) !}
$$

with $p=E / h \nu, q=D / h \nu, s=3 n-6$, where $\nu$ is the characteristic vibrational frequency of the cluster, $n$ is the number of 
atoms in the cluster, and $g$ is the reaction path degeneracy (the number of equivalent ways the reaction can occur).

Using the approach described above, a value of the dissociation energy can be deduced from the total internal energy required to dissociate the cluster on the experimental time scale. However, as pointed out in the Introduction, dissociation occurs from the liquidlike state of the cluster. The total internal energy of the liquidlike cluster $E$ is

$$
E=E_{V}+E_{C}-L^{0}(n),
$$

where $E_{V}$ is the initial vibrational energy in the solidlike cluster at $300 \mathrm{~K}$ and $E_{C}$ is the internal energy added by the collisions. The fraction of the ions translational energy converted into internal energy is obtained from an impulsive collision model. ${ }^{20}$ Note that $E_{V}$ and $E_{C}$ have distributions associated with them. We account for the distribution of initial vibrational energies using Eq. (3) but use an average value for $E_{C}$. The $E_{C}$ distribution is quite narrow because of the averaging inherent in the multicollision excitation process. ${ }^{20} L^{0}(n)$ in Eq. (5) is the energy difference between the solidlike and liquidlike clusters at $0 \mathrm{~K}$ (see Fig. 1),

$$
L^{0}(n)=L(n)+\Delta E(n),
$$

where $\Delta E(n)$ is the difference between the internal energies of the solidlike and liquidlike clusters at the melting temperature. A cluster does not have a melting point but melts over a range of temperatures. Assuming that we are in dynamic coexistence regime, where the transitions are between fully liquidlike and fully solidlike clusters, ${ }^{25-28}$ the melting temperature is defined as the temperature where the number of liquidlike clusters present in an ensemble equals the number of solidlike.

The dissociation of a liquidlike cluster could yield an $(n-1)$ atom product that is liquidlike or solidlike. Referring to Fig. 1, a process where a liquidlike cluster evaporates an atom and the product cluster simultaneously freezes is lower in energy than a process where the liquidlike cluster dissociates to form a liquidlike product (because of the latent heat released on freezing the product cluster). However, both dissociation and freezing are relatively slow processes (i.e., unlikely events) and the probability that both occur simultaneously is likely to be low. Formation of a solidlike product might occur, for example, in a true threshold measurement (which determines the minimum energy required for dissociation).

In order for a solidlike cluster product to form, enough energy must be removed during dissociation for the product cluster to freeze. Assuming a classical heat capacity, evaporating a single atom from the cluster will lower the temperature $^{29}$ of the product by approximately

$$
\Delta T=\frac{D_{L}+R T}{\left(3 n-\frac{15}{2}\right) R},
$$

where $n$ is the number of atoms in the parent cluster. Using the $D_{L}$ values determined below, $\Delta T$ ranges from $509 \mathrm{~K}$ for $\mathrm{Al}_{25}{ }^{+}$to $150 \mathrm{~K}$ for $\mathrm{Al}_{83}{ }^{+}$. The temperature of the evaporating clusters can be estimated from the measured dissociation thresholds to be $>2000 \mathrm{~K}$ (assuming a classical heat capacity). ${ }^{30}$ So, in the worst case, the temperature after

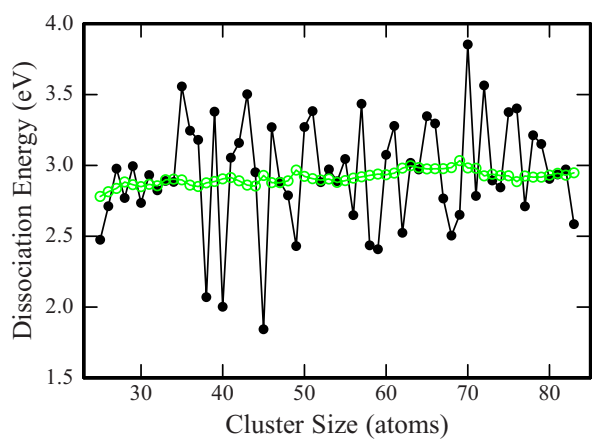

FIG. 2. (Color online) Plot of the dissociation energies of the liquidlike and solidlike clusters obtained from the analysis described in the text. The unfilled green circles show the $D_{L}$ values (for dissociation from the liquidlike cluster to give liquidlike products) and the filled black circles show the $D_{S}$ values (for dissociation from the solidlike cluster to give solidlike products). The $D_{L}$ values are obtained directly from analysis of the measured dissociation thresholds, the $D_{S}$ values result from adding to the $D_{L}$ values the contribution from the latent heats of the reactants and products.

evaporating one atom is still $>1500 \mathrm{~K}$. This is well above all of the melting temperatures for the $\mathrm{Al}_{n}^{+}$clusters with $n$ $=25-83$, which average around $650 \mathrm{~K}$. So, for the clusters studied here, dissociation must occur from the liquidlike state to yield liquidlike products.

The dissociation energies of the liquidlike clusters are obtained by fitting the measured average dissociation thresholds with Eq. (3). For $g$ in Eq. (4) we use $n^{2 / 3}$ (which is approximately the number of surface atoms) and for $\nu$ we use the Debye frequency of bulk aluminum. The fraction that dissociates is relatively insensitive to the value of $\nu$. For Eq. (6), a value for $L(n)$ is available from the previously reported heat capacity measurements for aluminum clusters. ${ }^{19} \Delta E(n)$ is expected to be small, although not entirely negligible. In the case of bulk aluminum, the difference is around $-0.008 \mathrm{eV} /$ atom. $^{31}$ Since we lack a reliable estimate for $\Delta E(n)$ we set $L(n)=L^{0}(n)$. The dissociation energies obtained from this analysis are shown as the unfilled green circles in Fig. 2. These dissociation energies are for the dissociation of liquidlike clusters to give liquidlike products $\left(D_{L}\right.$ in Fig. 1). The $D_{L}$ values show only small fluctuations with cluster size over the $n=25-83$ range examined here.

The relationship between the dissociation energy of the solidlike cluster $D_{S}$ and $D_{L}$ is

$$
D_{S}=D_{L}+L^{0}(n)-L^{0}(n-1) .
$$

The origin of the $L^{0}(n)$ term is evident from Fig. 1; it is the energy difference between the liquidlike and solidlike $n$ atom cluster at $0 \mathrm{~K}$. The $L^{0}(n-1)$ term results because the $n-1$ atom product is liquidlike, and the dissociation energy for the solidlike cluster $D_{S}$ is for the reaction $\mathrm{Al}_{n}^{+}(s) \rightarrow \mathrm{Al}_{n-1}{ }^{+}(s)$ $+\mathrm{Al}(g)$, where the product is solidlike. $L^{0}(n)$ and $L^{0}(n-1)$ are given by $L^{0}(n)=L(n)+\Delta E(n)$ and $L^{0}(n-1)=L(n-1)$ $+\Delta E(n-1)$, respectively. We lack reliable estimates for $\Delta E(n)$ and $\Delta E(n-1)$, since they are expected to be small and largely cancel, we set $L^{0}(n)=L(n)$ and $L^{0}(n-1)=L(n-1)$.

The filled black points in Fig. 2 show $D_{S}$ values determined using the prescription described here. The $D_{L}$ values were obtained by analyzing the measured dissociation thresholds (see above), and the latent heats were taken from 


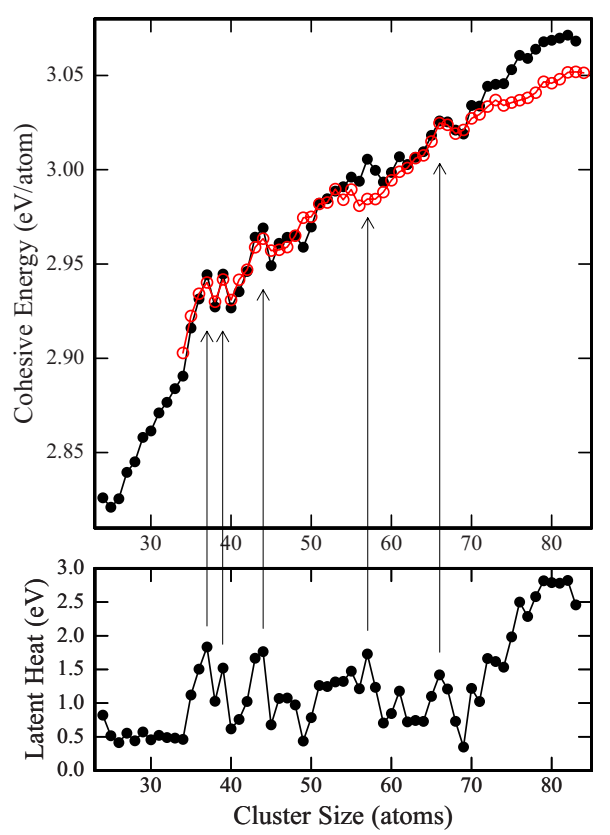

FIG. 3. (Color online) The upper panel shows the cohesive energies for solidlike $\mathrm{Al}_{n}{ }^{+}$clusters obtained from the analysis described in the text. The filled black points are the values determined from the experimental measurements. The unfilled red points show cohesive energies determined with density-functional theory for the lowest energy structures that were found for each cluster size in the calculations. The lower panel shows the latent heat per cluster plotted against cluster size (from Ref. 19).

previous heat capacity measurements (where the heat capacity, the derivative of the internal energy with temperature, is obtained by monitoring the change in the dissociation threshold with temperature). ${ }^{19}$ The $D_{S}$ values show much larger fluctuations than the $D_{L}$ values. There are substantial minima in the $D_{S}$ values at $n=38,40$, and 45 . The minima occur when the latent heat for the $n$ atom cluster is smaller than that for the $n-1$. Peaks in the $D_{S}$ values occur when the latent heat for the $n$ atom cluster is larger than that for the $n-1$; for example, $n=35,39$, and 43 .

The cluster cohesive energies are given by

$$
\begin{aligned}
C_{S}(n)=\frac{\sum_{n} D_{S}(n)}{n} & =\frac{\sum_{n}\left[D_{L}(n)+L^{0}(n)-L^{0}(n-1)\right]}{n} \\
& \approx \frac{L(n)+\sum_{n} D_{L}(n)}{n}
\end{aligned}
$$

Since the dissociation energies of the liquidlike clusters change smoothly with $n$, the variation in the cohesive energies of the solidlike clusters will track the variations in the latent heats. The cohesive energies are shown in Fig. 3 as the filled black points. The latent heats, determined from heat capacity measurements, ${ }^{19}$ are shown in the lower panel of Fig. 3. Local maxima in the cohesive energies are correlated with peaks in the latent heats.

The unfilled red circles in Fig. 3 show cohesive energies derived from the lowest energy structures found in calculations (see below). In the experimental work reported here, dissociation energies were determined for aluminum cluster cations with 25-83 atoms, and so in order to convert the measured dissociation energies into cohesive energies, it is necessary to assume a value for the cohesive energy of $\mathrm{Al}_{24}{ }^{+}$ (because an experimental value is not available). For the results shown in Fig. 3 we chose a value that led to the best overlap with the calculated cohesive energies.

\section{THE SEARCH FOR GLOBAL MINIMA}

Calculations were performed at the Kohn-Sham density-functional-theory ${ }^{32}$ level to search for the global minimum structures of $\mathrm{Al}_{n}{ }^{+}$cations with $n=34-84$. We consider this size range because the latent heats for $n=24-33$ are almost size independent, and the cohesive energies increase smoothly with cluster size. We employ the SIESTA code, ${ }^{33}$ with exchange and correlation effects treated within the spin-polarized generalized gradient approximation, ${ }^{34}$ and norm-conserving pseudopotentials to describe the core electrons. ${ }^{35,36}$ The basis set employed to expand the cluster wave function contains five basis functions per Al atom (a double zeta plus polarization or DZP basis in the standard notation $^{33}$ ). The spatial extension of the basis functions is determined by an energy shift ${ }^{33}$ of $20 \mathrm{meV}$. The fast-Fouriertransform mesh employed to evaluate some terms in the Hamiltonian is determined by a mesh cutoff ${ }^{33}$ of 100 Ry. The quality of the chosen basis set and pseudopotential was tested by performing calculations for the $\mathrm{Al}_{2}$ molecule and bulk aluminum, with results in good agreement with experiment and previous calculations at the same level of theory. ${ }^{37}$

The ideal search for global minima would employ an unbiased search method (such as a genetic ${ }^{38}$ or basin hopping algorithm ${ }^{39}$ ) in conjunction with an ab initio evaluation of the cluster energy. Unfortunately, this is still impractical for the size range we are considering. Therefore, the search for global minima was planned as a multistage task. In a first stage, we generated a sufficiently diverse set of initial structures for each cluster size. These structures are either taken directly from existing databases, ${ }^{40}$ explicitly built by considering typical icosahedral, decahedral, and octahedral atomic packings, or taken from finite temperature simulations performed with an approximate, orbital-free, energy functional. ${ }^{41}$ These trial structures include, in particular, the global minima of different potential models not necessarily mimicking aluminum clusters. In this sense, we are employing the system comparison approach advocated by Johnston and coworkers. ${ }^{38}$ In a second stage, these structures are fully optimized with the SIESTA code. The different structural families usually have quite different energies already at this stage, so some of them (for example, all the icosahedral clusters) can be excluded from further consideration. In a last and most computationally expensive stage, we take the five to ten more stable structures for each size, and consider them as seeds for further refinement. From each cluster of size $n$, we build $n+m$ and $n-m$ clusters (with $m=1-5$ ) by adding or removing atoms from its surface in many different ways. Each time we identify a better minimum for a given cluster size, it is considered the seed for a new refinement cycle. The procedure is possibly as systematic as it can be without the explicit employment of unbiased algorithms, and the number of different isomers tried for each size is about 300. The success of the global minima search will be demonstrated by the explicit comparison to the experimental results. 


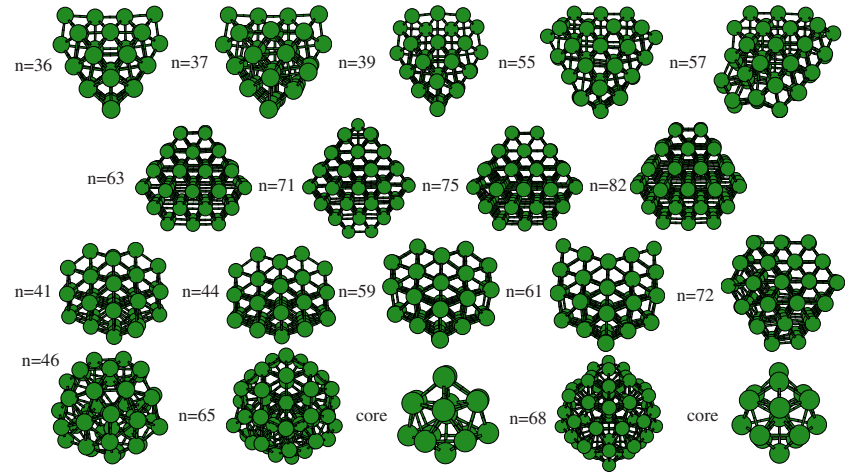

FIG. 4. (Color online) A selection of cluster structures. The different rows, from top to bottom, show examples of distorted decahedral fragments, perfect fcc, fcc with stacking faults (SFs), and disordered polytetrahedral isomers, respectively. The number of atoms is shown in the left side of each structure. For two of the disordered structures, we also show the first coordination shell of the innermost atom.

A representative selection of cluster structures is shown in Fig. 4, and the atomic coordinates for the putative global minima will be made available through the internet. ${ }^{42}$ The structures can be grouped into four different types which prevail over different size ranges: Distorted decahedral fragments, fragments of a face-centered-cubic (fcc) lattice, fcc fragments with stacking faults (SFs), and other "disordered" rounded structures characterized by a crowded cluster core (the central atom is coordinated to 15 neighbors) and a wider distribution of bond lengths. Examples of these four types of structure are shown in different rows of Fig. 4.

The atoms in the distorted decahedral family do not grow symmetrically around the fivefold decahedral axis, which is located close to the cluster surface. Figure 5 shows schematically how these distorted decahedra are obtained by removing atoms from a perfect parent decahedron. The parent decahedron is of a special kind as it does not expose any noncompact (100) facets, so it is a pentagonal bipyramid.

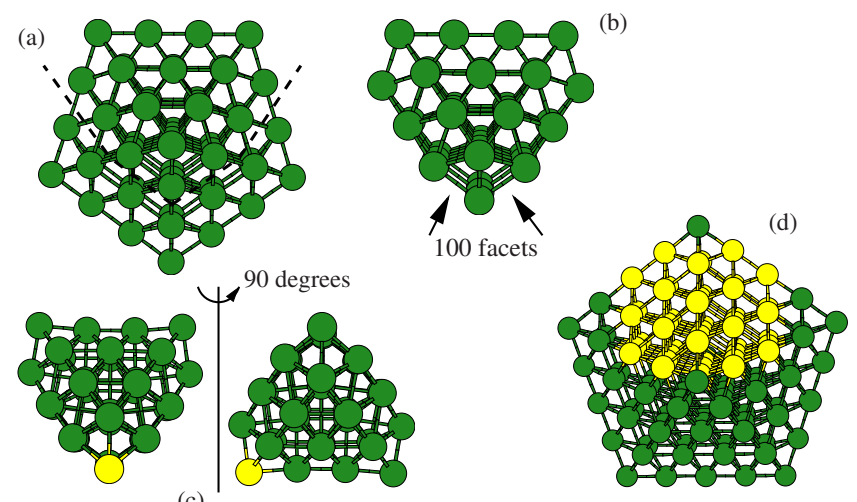

FIG. 5. (Color online) (a) A perfect 54-atom decahedron. The thick dashed line shows the atoms that must be removed in order to obtain a 35 -atom decahedral fragment, which exposes two energetically unfavorable (100) facets, shown with arrows in (b). Adding one more atom (yellow or light gray) to the bottom row of atoms and displacing this row removes the (100) facets and results in the perfect 36-atom cluster shown in (c). Further distortion creates an additional fivefold axis as demonstrated in the rotated view. This cluster exposes only (111) compact facets. (d) shows how the 44-atom cluster can also be considered an undistorted fragment of the same 54-atom parent decahedron. This cluster does not preserve the fivefold axis and belongs therefore to the SF family.
The resulting decahedral fragment exposes two (100) facets, which is energetically unfavorable for aluminum clusters (see below). Then, the displacement of a full row of atoms results in a perfect 36-atom cluster, which only exposes compact (111) facets. The distorted decahedra are the global minima for cluster sizes around $n=36$ and $n=55$. Similar larger structures can be built around $n=76$ but they are not the global minima at this point.

For most other sizes, pure fcc and SF structures dominate. Like the distorted decahedra, a large fraction of the surface exposed by these structures has compact, (111)-like surface terminations. In fact, for most sizes it is possible to devise more compact fcc and SF structures (with more interior atoms), but they have a higher energy because of a relatively large proportion of (100) facets. However, for $n$ $=77-84$ a larger proportion of (100) facets is obtained. This might imply that simpler packing effects determine the optimal structures in this size range, although the poor agreement with experiment for these large sizes (see Fig. 3 and explanation below) suggests that more stable geometries, exposing mostly (111) facets, may not have been found. Rounded disordered structures only occur close to the spherical electron shell closings predicted by the jellium model at 138 and 198 electrons (see below).

\section{DISCUSSION}

\section{Comparison with previous theoretical studies}

The parametrized model potentials usually employed to describe aluminum (embedded atom, glue and similar models) predict the structures to be either icosahedral ${ }^{43}$ or polytetrahedral, ${ }^{44}$ and locate the transition to bulklike fcc fragments at significantly larger sizes than examined here. Our $a b$ initio results clearly show that icosahedral isomers have very high energies; roughly spherical polytetrahedral isomers are more stable than icosahedra for all sizes but they are the global minima only close to the spherical electron shell closings. Thus, our calculations demonstrate that the present parametrizations of aluminum potential models should be revised. Improved interatomic potentials should more strongly penalize the bond strain typical of icosahedra.

Aguado and López ${ }^{45}$ published a preliminary report on the $a b$ initio structures of $\mathrm{Al}_{n}{ }^{+}$clusters with $n=46-62$. Although the main structural trends [the preponderance of structures with low strain and a high proportion of (111) facets] were identified there, the global minima reported here for most sizes with $n=46-62$ are significantly lower in energy. Only the structures identified here lead to cohesive energies in good agreement with experiment (see below). Moreover, in the present work, we significantly enlarge the size range covered by the calculations. Kanhere and collaborators recently published putative global minima for a few selected sizes using the VASP code. ${ }^{46}$ The global minima presented here have lower energies (both in SIESTA and VASP calculations ${ }^{47}$ ) than the structures suggested by Kanhere for all sizes except $n=46$, for which we obtain similar disordered structures. Other $a b$ initio studies ${ }^{48}$ focused on a few selected sizes, and in some cases found evidence for the high stability of fcc structures. We have explicitly checked that 
the structures suggested in those studies are high-energy isomers according to the SIESTA calculations presented here.

\section{Comparison of measured and calculated cohesive energies}

The cohesive energies for the lowest energy geometry found for each cluster size are shown in Fig. 3 as the unfilled red circles. Most of the fluctuations observed in the measured cohesive energies are reproduced in the calculated values. Note in particular the maxima at 37, 39, 44, and 66 atoms which are evident in both the experimental values and the calculated cohesive energies. The maximum in the measured cohesive energies at 57 is not reproduced in the calculations, and there is a narrow range of cluster sizes (56-61) where the calculated cohesive energies are significantly below the measured values. It is possible that the lowest energy structures have not been found for these cluster sizes. Interestingly, for clusters with 56-62 atoms, there are "dips" or local minima ${ }^{18}$ in the heat capacities at temperatures just below the peaks in the heat capacities due to the melting transition. The dips, which are only observed for a few specific cluster sizes, have been attributed to annealing from a high-energy geometry to the ground state, ${ }^{18,49}$ and their presence may be related to a structural change.

For clusters with more than around 71 atoms, the calculated cohesive energies are significantly below the measured values. Again, it is possible that the lowest energy structures have not been identified in the calculations. As the cluster size increases, it becomes more difficult to find the lowest energy geometry, particularly if the structure changes significantly. There are dips in the heat capacities for clusters with 77-83 atoms, which again seem to be consistent with an annealing transition and hence a structural change in this size regime. ${ }^{22,49}$ The discrepancy between theory and experiment in this size regime is sufficiently large that we believe the true ground states do not have pure fcc or SF structures, but probably have a structure with mixed symmetry.

For clusters with more than about 500 atoms, Martin et al. showed that aluminum clusters adopt fcc structures with an octahedral shape. ${ }^{50}$ Our calculations suggest that the fcclike structures (possibly with some stacking faults) are already energetically competitive for clusters with about 60 atoms. Nevertheless, the transition to fcc-like structures is far from complete within the size range considered in this work. We believe we are observing the initial part of the transition, which will probably occur over a broad size range.

For a few clusters in Fig. 3 (for example, $n=45$ and 49), it appears that the experimental cohesive energy (filled black circles) is significantly below the calculated value (unfilled red circles). In these cases there may be a problem with the experimental value. The peaks in the heat capacities of $n$ $=45$ and 49 are broad and low in intensity and it is plausible that the latent heat has been underestimated for these clusters.

\section{Factors controlling the stabilities of $\mathrm{Al}_{n}{ }^{+}$clusters}

The stabilities of metal clusters are influenced by both structural and electronic effects. We address first the struc-
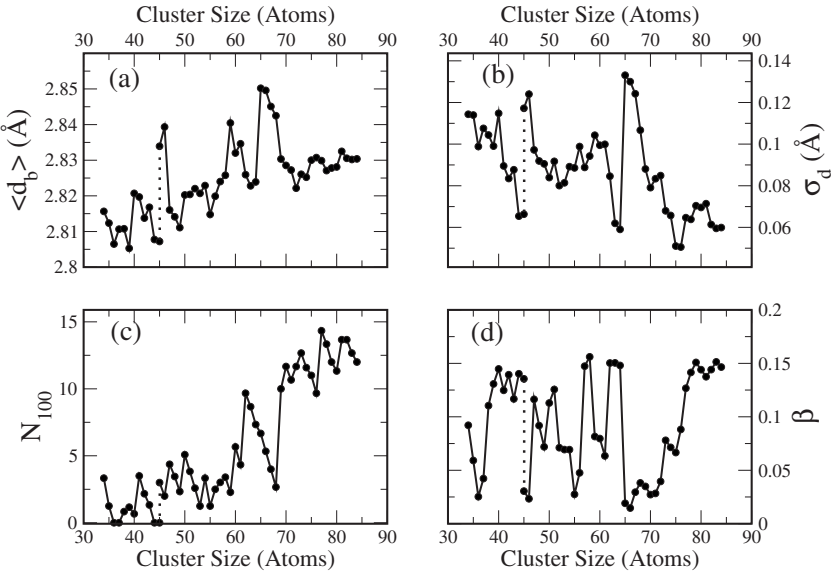

FIG. 6. Structural indicators for $\mathrm{Al}_{n}{ }^{+}$clusters from the calculations. (a) shows the average bond length, (b) its root-mean-squared deviation, (c) the number of atoms at (100) facets and (d) the asphericity shape parameter, for the global minimum structures found in this work. For $\mathrm{Al}_{45}{ }^{+}$, there is one disordered isomer nearly degenerate with the ordered ground state, so we quote the structural indicators for both isomers, joined with a dotted line.

tural stability, which is determined by several competing factors. Based on the observed structural trends, we choose the following indicators.

(a) The average bond distance $\left\langle d_{b}\right\rangle$. Shorter and stronger bonds are usually associated with more stable structures. To calculate the average bond distance, we assume that two atoms have a bond when their separation is shorter than a cutoff distance. The cutoff is chosen to be $10 \%$ longer than the bulk bond distance, although the observed trends are not very sensitive to small variations in the precise value.

(b) The root-mean-squared deviation from the average bond length $\sigma_{d}=\left(\left\langle d_{b}^{2}\right\rangle-\left\langle d_{b}\right\rangle^{2}\right)^{1 / 2}$. Our results suggest that bond strain is significantly penalized in aluminum clusters. A larger bond strain will result in a larger $\sigma_{d}$ value.

(c) The number of surface atoms contributing to noncompact (100) facets, $N_{100}$. When a given atom belongs to several surface facets, we count the appropriate fraction. For example, for one atom lying on a corner site joining two (111) and one (100) facets, we count onethird of an atom on the (100) facet. This way we have an operational measure for $N_{100}$ which is simple to evaluate.

(d) The Hill-Wheeler asphericity parameter $\beta .^{51}$ This parameter is evaluated from the inertia tensor and is exactly zero for a spherical cluster. The larger $\beta$, the less spherical the cluster shape. This shape parameter is interesting in connection with the electronic stabilities shown below.

Plots of these four structural indicators are shown in Fig. 6 . For $n=45$, there is one disordered isomer which is nearly degenerate with the ordered ground state and we show the structural indicators for both isomers. Regarding the average bond length [Fig. 6(a)], there are clear local minima at $n$ $=36,39,44,49$, and 55, and important local maxima for the disordered isomers around 46 and 66 atoms (hence the two 


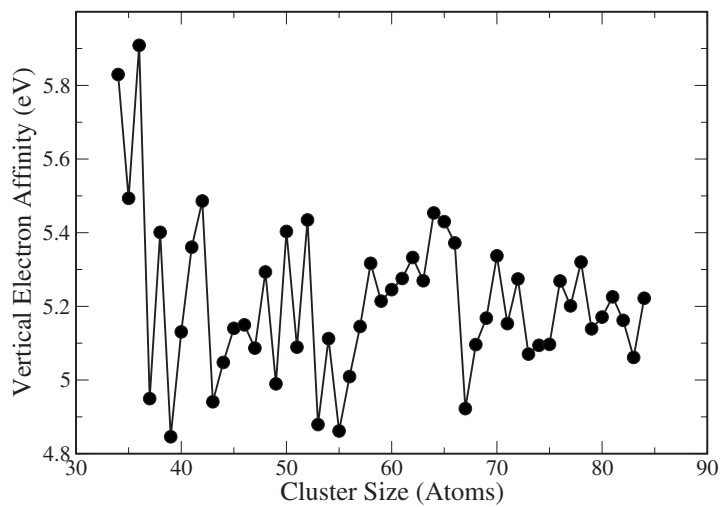

FIG. 7. Vertical electron affinities (VEAs) of $\mathrm{Al}_{n}{ }^{+}$clusters, calculated for each size as the total energy difference between the cation and the neutral cluster at the optimal geometry of the cation.

very different values for $n=45)$. The bond length dispersion [Fig. 6(b)] has local maxima for the disordered structures, showing that those structures sustain significant bond strain. For the rest of the structures, it consistently shows the smallest values for fcc clusters ( $n=62-63$ and the largest sizes), and intermediate values for SF and distorted decahedral clusters. On top of this general trend, there are clear local minima at $n=36,39$, and 44. $N_{100}$ [Fig. 6(c)] is exactly zero for $n=36,37$, and 44 (for $n=45$ it depends on the isomer), and has significant local minima at $n=53,55$, and 68. Finally, the most spherical clusters [local minima in Fig. 6(d)] are $n=36,46,55,65$, and 66 . All these sizes are close to the observed electron shell closings for the cations or to the expected electron shell closings of neutral aluminum clusters (see below).

Overall, $\mathrm{Al}_{36}{ }^{+}$and $\mathrm{Al}_{44}{ }^{+}$satisfy all the structural stability requirements and can be considered as geometrical "shell closings." They have short and strong bonds, little strain compared to their immediate neighbors, and perfect (111) surface terminations. $\mathrm{Al}_{55}{ }^{+}$also has short bond lengths and a low proportion of (100) facets, but the bond strain is not particularly small; $\mathrm{Al}_{39}{ }^{+}$has a short average bond length and shows a local minimum in the bond strain but exposes a relatively large proportion of (100) facets as compared to their neighbors. Therefore, $n=39$ and 55 should also have an enhanced structural stability but lower than $n=36$ and 44 .

\section{Contribution of electronic shell closings to stability of $\mathrm{Al}_{n}^{+}$}

Figure 7 shows the calculated vertical electron affinity (VEA). This quantity is defined as the total energy difference between the cluster cation and the neutral cluster, both clusters being at the optimal geometry of the cation. It can be used as a measure of the electron shell contribution to cluster stability. ${ }^{52}$ The clusters showing an enhanced electron shell stability (particularly low VEA values) are $n=37,39,43,53$, 55, and 67. Schriver et al. ${ }^{53}$ measured the vertical ionization energy (VIE) of neutral aluminum clusters in the size range relevant to this study. The VIE is the total energy difference between the neutral cluster and the cluster cation, both clusters being at the optimal geometry of the neutral. If the global minimum geometries of the neutral clusters are the same as those of the cations studied here, then the VIE of the neutrals and the VEA of the cations should be correlated. Schriver et al. found electron shell openings (particularly low VIE values) for neutral $\mathrm{Al}_{n}$ clusters with $n=37,39,43$, 47,55 , and 67 , in good agreement with our theoretical results. Additional calculations for neutral clusters ${ }^{54}$ show that the cation and neutral lowest energy structures are not the same for $n=47$, and confirm the experimentally observed shell opening for neutral $\mathrm{Al}_{47}$, as well as the expected spherical shell closings for neutral $\mathrm{Al}_{46}$ (138 electrons) and $\mathrm{Al}_{66}$ (198 electrons). Thus, the theoretically determined cluster structures not only reproduce the measured cohesive energies but also fully explain (for the first time to the best of our knowledge) the photoionization experiments of Schriver et $a l .{ }^{53}$ This gives indirect support to the strategy adopted to locate the global minima.

Many of the observed electron shell closings cannot be explained by the spherical jellium model or by its ellipsoidally distorted extensions. This was already noticed in the experimental work of Schriver et al.: "Although there is clear evidence of electron shell filling, substantial deviations from shell model predictions remain." ${ }^{, 53}$ At the time when this experimental work was published, there was such a strong expectation that the structure of aluminum clusters should be icosahedral, that Schriver et al. suggested an interpretation of their results in terms of the crystal-field splitting of high angular momentum electron levels in icosahedral clusters. The present calculations show that crystal-field effects, as determined by the precise location of the aluminum atoms, are certainly very important but the structures are not icosahedral. The complex interplay between the atomic structure and the spectrum of electron energy levels suggests that devising accurate potential models for aluminum clusters of this size will be a very complicated task.

\section{Size evolution of cohesive energies and latent heats}

The calculations demonstrate that the size evolution of the cohesive energies (and therefore of the latent heats) results from the combined action of electronic and structural effects. $\mathrm{Al}_{37}{ }^{+}$is not particularly stable from the structural point of view, as it contains one atom with low coordination on top of the 36-atom geometrical shell closing but it is strongly stabilized by an electronic shell closing. Preliminary results for neutral clusters ${ }^{54}$ show that $\mathrm{Al}_{36}$ is both a geometrical and an electronic shell closing and, in fact, the enhancement of the cohesive energy of $\mathrm{Al}_{36}$ in the neutral series is bigger than for the 37-atom cluster in the cation series. $\mathrm{Al}_{66}{ }^{+}$and $\mathrm{Al}_{67}{ }^{+}$are similar cases: Here the electron shell closing stabilizes highly strained but spherical structures which would be otherwise quite unstable. An opposite example is $\mathrm{Al}_{44}{ }^{+}$, whose enhanced stability is due only to the structural effects, as its VEA is not particularly small. Finally, the enhanced stabilities of $n=39,43$, and 55 are due to the combined structural and electronic effects.

Extensive and systematic experimental results on the melting properties of unsupported clusters are still scarce, so a comparison with the other paradigm (sodium clusters) is in order here. The experiments of Haberland and 


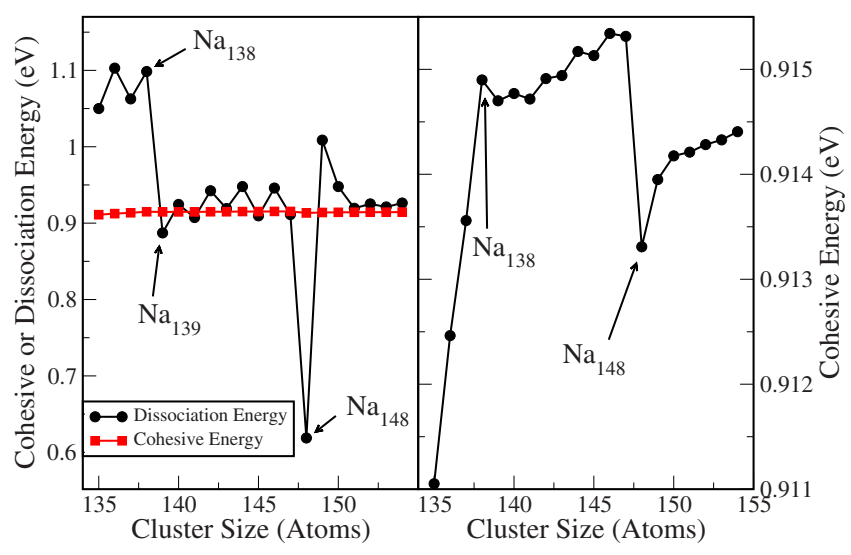

FIG. 8. (Color online) The left panel shows a comparison of dissociation and cohesive energies for neutral sodium clusters with 135-154 atoms. Note that the dissociation energy of $\mathrm{Na}_{148}$ is substantially lower than the cohesive energy of $\mathrm{Na}_{147}$. The right panel shows a blowup of the cohesive energies. A significant drop only occurs between 147 and 148 atoms.

co-workers ${ }^{13-15,55}$ and the $a b$ initio molecular dynamics simulations of Aguado and co-workers ${ }^{56}$ demonstrated that structural effects alone can explain the size dependence of the latent heats of sodium clusters. This is in marked contrast with the aluminum clusters studied here, where the enhanced electron stabilities significantly affect the latent heats of melting. Due to a stronger electron-ion interaction in aluminum, the gaps separating electron energy levels are much larger than in Na clusters of a similar size. In fact, discontinuities in the VIE values at the electron shell closings for aluminum are about four times larger than for the alkali metals. It is therefore not surprising that electron shell closings have a stronger effect on the thermal properties of aluminum clusters than on sodium clusters of similar size. According to this view, electron shell effects might also be visible in the melting properties of sodium clusters of sufficiently small size, where the quantum confinement effect leads to large gaps in the electron energy level spectrum. Ghazi et al. ${ }^{57}$ have recently found a significant influence of the 40-electron shell closing on the melting temperatures of Na clusters with 39-41 atoms, although they do not provide the associated latent heats.

In order to show the generality of the correlation between cohesive energies and latent heats, we test it explicitly here for sodium clusters $\mathrm{Na}_{n}$ with $n=135-154$ atoms. In this size range, an electron shell closing occurs for $n=138$ and an icosahedral structural shell closing is observed at $n=147$, but a local maximum in the latent heat is observed only for the geometrical shell closing. ${ }^{55,56}$ We have optimized the structures of these clusters with the SIESTA code using as starting structures those provided by the previous orbital-free calculations. ${ }^{56}$ Figure 8 shows the calculated dissociation and cohesive energies. The dissociation energy curve shows two large drops, one after the electronic shell closing and the other one after the icosahedral shell closing. In the evaporative ensemble realized in typical mass abundance experiments, only the electron shell closing survives because the sodium clusters are sufficiently hot that the atoms evaporate from liquidlike clusters.

When one atom is added to the $(n-1)$-atom cluster, the cohesive energy will increase if and only if $D_{S}(n)>C_{S}(n$ $-1)$. This is easily demonstrated using the relationship between the cohesive and dissociation energies [Eq. (9)]. Right after the electron shell closing at $n=138$, the dissociation energies remain, on average, larger than the cohesive energies. $D_{S}(139)$ is only slightly smaller than $C_{S}(138)$, and thus the induced local maximum in the cohesive energy at $n$ $=138$ is not very important (in fact, it is of a similar order of magnitude as the odd-even oscillations seen between $n$ $=130$ and $n=147$ ). In other words, the electron shell closing is not sufficiently strong to induce a marked local maximum in the cohesive energy curve. $D_{S}(148)$, on the other hand, is substantially smaller than $C_{S}(147)$, which induces the only large drop in the cohesive energy curve. The atom added to the perfect three-shell icosahedron is very easily dissociated. The cohesive energy curve shown in Fig. 8 reproduces the main trends in the experimental latent heats for this size range: A rapid increase up to the electron shell closing, then a more steady increase between the electron and the structural shell closings, and finally a large drop after the structural shell closing. Therefore, the correlation between the latent heats and cohesive energies holds both for aluminum and sodium clusters, but the relative weight of electron shell closings is smaller in sodium clusters.

More generally, whenever the electron shell contribution to cluster stability is significantly different in solidlike and liquidlike phases, electronic effects must affect the melting properties. For example, a solidlike cluster may have a large highest occupied molecular orbital-lowest unoccupied molecular orbital (HOMO-LUMO) gap which is significantly decreased upon melting, so the liquidlike cluster is much less stable from the electronic point of view. Such a difference in electronic stability of the two phases will then be reflected in the latent heat of melting. This is, in fact, what we observe for aluminum clusters (see Fig. 2). In the case of sodium clusters, Aguado and López ${ }^{56}$ checked a few sizes and found that the HOMO-LUMO gaps were similar in the solidlike and liquidlike clusters (although slightly smaller in the liquidlike phase). The slight cohesive energy stabilization induced by the electron shell closing for the 138-electron solidlike cluster (see Fig. 8) is then of the same order of magnitude as the corresponding stabilization of the liquidlike cluster. Furthermore, the gaps are significantly smaller than the latent heats per atom, so the electronic effect on the melting properties is small. We are presently undertaking $a b$ initio simulations of liquid aluminum clusters to check the corresponding situation in simple trivalent metals.

\section{CONCLUSIONS}

In this manuscript we have outlined the connections between the latent heats and cohesive energies of metal clusters. The cohesive energies of solidlike clusters are given by

$$
C_{S}(n) \approx \frac{L(n)+\sum_{n} D_{L}}{n} .
$$

Since $\Sigma D_{L}$ shows only small fluctuations with cluster size, the size-dependent variations in the cohesive energies are due almost entirely to the size-dependent variations in the 
latent heats. The cohesive energies of solidlike $\mathrm{Al}_{n}{ }^{+}$clusters determined from the measured latent heats and the $D_{L}$ values closely track cohesive energies determined for the lowest energy geometry $\mathrm{Al}_{n}^{+}$found with density-functional theory. In most cases where theory and experiment depart, it is likely that the ground state has not been found in the calculations. The calculations also account for many of the features in the measured ionization energies of $\mathrm{Al}_{n}$ clusters.

The lowest energy structures found in the calculations are characterized by low strain and a high proportion of (111) facets. They can be grouped into four different types which prevail over different size ranges: Distorted decahedral fragments, fcc fragments, fcc fragments with SFs, and disordered rounded structures. Icosahedral geometries are high-energy isomers and we suggest that present aluminum potentials should be revised to more strongly penalize the bond strain typical of icosahedra. According to the calculations, the size evolution of the cohesive energies (and therefore of the latent heats) results from a combination of electronic and structural effects. For some clusters (e.g., $\mathrm{Al}_{37}{ }^{+}$and $\mathrm{Al}_{66}{ }^{+}$), an electronic shell closing causes a maximum in the cohesive energy and the latent heat, while for others (e.g., $\mathrm{Al}_{44}{ }^{+}$), a structural shell closing is responsible. For $\mathrm{Al}_{66}{ }^{+}$, the electronic shell closing stabilizes a highly strained but spherical geometry.

Our calculations show that the correlation between cohesive energies and latent heats also holds for sodium clusters, which demonstrates the generality of this effect. For the size range covered in the calorimetric experiments on sodium clusters, however, the energy stabilization associated with the electronic shell closings is not sufficiently strong to induce significant local maxima in the cohesive energies of solidlike clusters, so structural effects alone can explain the main trends in the size evolution of the latent heat.

The strong correlation between the cohesive energies and latent heats observed in this work is expected to hold quite generally, as long as the two following conditions are met: (1) The size evolution of the dissociation energies of liquidlike clusters should be much smoother than that for the solidlike clusters; (2) there should not be a premelting transition (such as a solid-solid isomerization transition) involving a large latent heat. In those cases, Eq. (10) should be generalized to

$$
C_{S}(n)=\frac{L(n)+L_{S-S}(n)+\sum_{n} D_{L}}{n},
$$

where $L_{S-S}(n)$ is the latent heat absorbed at the solid-solid transition.

\section{ACKNOWLEDGMENTS}

We gratefully acknowledge the support of the National Science Foundation, the Spanish MEC, and the European Regional Development Fund (Projects Nos. MAT200503415 and VA068A06).

${ }^{1}$ C. E. Klots, J. Phys. Chem. 92, 5864 (1988).

${ }^{2}$ C. Brechignac, P. Cahuzac, J. Leygnier, and J. Weiner, J. Chem. Phys. 90, 1492 (1989).

${ }^{3}$ U. Ray, M. F. Jarrold, J. E. Bower, and J. S. Kraus, J. Chem. Phys. 91,
2912 (1989).

${ }^{4}$ P. P. Radi, M. T. Hsu, J. Brodbelt-Lustig, M. Rincon, and M. T. Bowers, J. Chem. Phys. 92, 4817 (1990).

${ }^{5}$ D. A. Hales, L. Lian, and P. B. Armentrout, Int. J. Mass Spectrom. Ion Process. 102, 269 (1990).

${ }^{6}$ C. Brechignac, P. Cahuzac, F. Carlier, M. Defrutos, and J. Leygnier, J. Chem. Phys. 93, 7449 (1990).

${ }^{7}$ C. E. Klots, Z. Phys. D: At., Mol. Clusters 21, 335 (1991).

${ }^{8}$ L. Lian, C. X. Su, and P. B. Armentrout, J. Chem. Phys. 97, 4084 (1992).

${ }^{9}$ C. Brechignac, P. Cahuzac, N. Kebaili, and J. Leygnier, J. Chem. Phys. 112, 10197 (2000).

${ }^{10}$ C. L. Briant and J. J. Burton, J. Chem. Phys. 63, 2045 (1975).

${ }^{11}$ R. D. Etters and J. Kaelberer, J. Chem. Phys. 66, 5112 (1977).

${ }^{12}$ R. S. Berry, J. Jellinek, and G. Natanson, Chem. Phys. Lett. 107, 227 (1984).

${ }^{13}$ M. Schmidt, R. Kusche, W. Kronmüller, B. von Issendorf, and H. Haberland, Phys. Rev. Lett. 79, 99 (1997).

${ }^{14}$ M. Schmidt, R. Kusche, B. von Issendorf, and H. Haberland, Nature (London) 393, 238 (1998).

${ }^{15}$ M. Schmidt and H. Haberland, C. R. Phys. 3, 327 (2002).

${ }^{16}$ G. A. Breaux, R. C. Benirschke, T. Sugai, B. S. Kinnear, and M. F. Jarrold, Phys. Rev. Lett. 91, 215508 (2003).

${ }^{17}$ G. A. Breaux, D. A. Hillman, C. M. Neal, R. C. Benirschke, and M. F. Jarrold, J. Am. Chem. Soc. 126, 8628 (2004).

${ }^{18}$ G. A. Breaux, C. M. Neal, B. Cao, and M. F. Jarrold, Phys. Rev. Lett. 94, 173401 (2005).

${ }^{19}$ C. M. Neal, A. K. Starace, and M. F. Jarrold, Phys. Rev. B 76, 054113 (2007).

${ }^{20}$ M. F. Jarrold and E. C. Honea, J. Chem. Phys. 95, 9181 (1991).

${ }^{21}$ J. M. Hunter, J. L. Fye, M. F. Jarrold, and J. E. Bower, Phys. Rev. Lett. 73, 2063 (1994).

${ }^{22}$ C. M. Neal, A. K. Starace, and M. F. Jarrold, J. Am. Soc. Mass Spectrom. 18, 74 (2007)

${ }^{23}$ C. M. Neal, G. A. Breaux, B. Cao, A. K. Starace, and M. F. Jarrold, Rev. Sci. Instrum. 78, 075108 (2007).

${ }^{24}$ O. K. Rice and H. C. Ramsperger, J. Am. Chem. Soc. 50, 617 (1928).

${ }^{25}$ R. S. Berry, J. Jellinek, and G. Natanson, Phys. Rev. A 30, 919 (1984).

${ }^{26}$ T. L. Beck, J. Jellinek, and R. S. Berry, J. Chem. Phys. 87, 545 (1987).

${ }^{27}$ J. P. Rose and R. S. Berry, J. Chem. Phys. 98, 3246 (1993).

${ }^{28}$ B. Vekhter and R. S. Berry, J. Chem. Phys. 106, 6456 (1997).

${ }^{29} \mathrm{We}$ use temperature here loosely, as defined by $T=E_{\text {int }} /((3 n-6+3 / 2) R)$. This is a reasonable approximation because the internal energy distribution is close to Gaussian with a standard deviation significantly less than the average.

${ }^{30}$ This is estimated from dissociation thresholds measured at $1073 \mathrm{~K}$. These measurements will be reported elsewhere.

${ }^{31}$ Based on the enthalpy differences between the liquid and the solid at the melting temperature and at $298 \mathrm{~K}$, determined from information on the NIST Chemistry WebBook (http://webbook.nist.gov/chemistry/).

${ }^{32}$ W. Kohn and L. J. Sham, Phys. Rev. 140, 1133 (1965).

${ }^{33}$ J. M. Soler, E. Artacho, J. D. Gale, A. García, J. Junquera, P. Ordejón, and D. Sánchez-Portal, J. Phys.: Condens. Matter 14, 2745 (2002).

${ }^{34}$ J. P. Perdew, K. Burke, and M. Ernzerhof, Phys. Rev. Lett. 77, 3865 (1996); 78, 1396 (1997).

${ }^{35}$ R. Hamann, M. Schlüter, and C. Chiang, Phys. Rev. Lett. 43, 1494 (1979).

${ }^{36}$ L. Kleinman and D. M. Bylander, Phys. Rev. Lett. 48, 1425 (1982).

${ }^{37}$ I. Lee and R. M. Martin, Phys. Rev. B 56, 7197 (1997).

${ }^{38}$ D. M. Deaven and K. M. Ho, Phys. Rev. Lett. 75, 288 (1995); L. O. Paz-Borbón, R. L. Johnston, G. Barcaro, and A. Fortunelly, J. Chem. Phys. 128, 134517 (2008).

${ }^{39}$ D. J. Wales and J. P. K. Doye, J. Phys. Chem. A 101, 5111 (1997).

${ }^{40}$ The Cambridge Cluster Database, D. J. Wales, J. P. K. Doye, A. Dullweber, M. P. Hodges, F. Y. Naumkin, F. Calvo, J. Hernández-Rojas, and T. F. Middleton (http://www-wales.ch.cam.ac.uk/CCD.html).

${ }^{41}$ A. Aguado, D. J. González, and J. M. López, in Trends in Chemical Physics Research, edited by A. N. Linke (Nova Science Publishers, Inc., New York, 2005), Chap. 10, pp. 205-269.

${ }^{42}$ Web page of the Nanometric Properties of Matter Research Group (http:// metodos.fam.cie.uva.es/ GIR).

${ }^{43}$ R. Werner, Eur. Phys. J. B 43, 47 (2005); E. G. Noya, J. P. K. Doye, and F. Calvo, Phys. Rev. B 73, 125407 (2006); S. Alavi and D. L. Thompson, J. Phys. Chem. A 110, 1518 (2006); W. Zhang, F. Zhang, and Z. Y. Zhu, Phys. Rev. B 74, 033412 (2006); Eur. Phys. J. D 43, 97 (2007). 
${ }^{44}$ D. Y. Sun and X. G. Gong, Phys. Rev. B 57, 4730 (1998); J. P. K. Doye, J. Chem. Phys. 119, 1136 (2003); J. Joswig and M. Springborg, Phys. Rev. B 68, 085408 (2003).

${ }^{45}$ A. Aguado and J. M. López, J. Phys. Chem. B 110, 14020 (2006).

${ }^{46}$ C. M. Neal, A. K. Starace, M. F. Jarrold, K. Joshi, S. Krishnamurty, and D. G. Kanhere, J. Phys. Chem. C 111, 17788 (2007).

${ }^{47}$ K. Joshi, personal communication (21 January 2008).

${ }^{48}$ H. P. Cheng, R. S. Berry, and R. L. Whetten, Phys. Rev. B 43, 10647 (1991); J. Yi, D. Oh, and J. Bernholc, Phys. Rev. Lett. 67, 1594 (1991); J. Akola, M. Manninen, H. Häkkinen, U. Landman, X. Li, and L. Wang, Phys. Rev. B 62, 13216 (2000); K. Manninen, J. Akola, and M. Manninen, ibid. 68, 235412 (2003); R. Fournier, J. Chem. Theory Comput. 3, 921 (2007); W. Zhang, W. Lu, J. Sun, C. Z. Wang, and K. M. Ho, Chem. Phys. Lett. 455, 232 (2008).

${ }^{49}$ M. F. Jarrold, B. Cao, A. K. Starace, C. M. Neal, and O. H. Judd, J. Chem. Phys. 129, 014503 (2008).

${ }^{50}$ T. P. Martin, U. Näher, and H. Schaber, Chem. Phys. Lett. 199, 470 (1992).
${ }^{51}$ A. Bulgac and D. Kusnezov, Phys. Rev. B 45, 1988 (1992).

${ }^{52}$ We prefer to show the VEA rather than the HOMO-LUMO gap because the latter quantity is not easily defined within a spin-polarized framework when the cluster has an odd number of electrons. For clusters with an even number of electrons, the electron shell closings predicted by the VEA (see Fig. 7) coincide with maximum values of the HOMO-LUMO gap, as they should.

${ }^{53}$ K. E. Schriver, J. L. Persson, E. C. Honea, and R. L. Whetten, Phys. Rev. Lett. 64, 2539 (1990).

${ }^{54}$ (Unpublished).

${ }^{55}$ H. Haberland, Th. Hippler, J. Donges, O. Kostko, M. Schmidt, and B. von Issendorff, Phys. Rev. Lett. 94, 035701 (2005).

${ }^{56}$ A. Aguado and J. M. López, Phys. Rev. Lett. 94, 233401 (2005); A. Aguado, J. Phys. Chem. B 109, 13043 (2005); E. G. Noya, J. P. K. Doye, D. J. Wales, and A. Aguado, Eur. Phys. J. D 43, 57 (2007).

${ }^{57}$ S. M. Ghazi, M. S. Lee, and D. G. Kanhere, J. Chem. Phys. 128, 104701 (2008). 